\title{
Long-Term Course and Clinical Characteristics of Ventricular Tachycardia Detected in Children by School-Based Heart Disease Screening
}

\author{
Mari Iwamoto, MD; Ichiroh Niimura, MD ; Toshimitsu Shibata, MD \\ Kiyoshi Yasui, MD*; Kiyohiro Takigiku, MD**; Takashi Nishizawa, MD*; \\ Tohru Akaike, MD; Shunpei Yokota, MD**
}

\begin{abstract}
Background The prognosis of ventricular tachycardia (VT) in children with overt heart disease is generally good, so the aim of this study was to review the prognosis and necessity of treatment of VT that detected by school-based heart disease screening.

Methods and Results Of the 48 cases of pediatric VT that have been followed for 2-30 years, $17 \%$ were diagnosed at the first school-based screening test (12-lead ECG at rest) and the remainder who had premature ventricular contractions (PVC) on the resting 12-lead ECG required Holter ECG test and exercise stress ECG test to detect VT. In $90 \%$ of cases, VT in healthy children is idiopathic non-sustained VT and more than half of the present cases showed natural disappearance of the VT during follow up. In particular, cases of monomorphic PVC or maximum PVC runs less than 5 had a good prognosis. The cases of polymorphic VT and VT with heart disease continued for the long term.

Conclusion Treatment is unnecessary for monomorphic VT with the maximum number of salvos less than 5 . The necessity for treatment depends on the symptoms and profile of the VT. The prognosis for polymorphic VT and underlying heart disease is not good and may require implantable cardioverter defibrillator. (Circ J 2005; 69: $273-276$ )
\end{abstract}

Key Words: Idiopathic ventricular tachycardia; Polymorphic ventricular tachycardia; School heart disease screening

$\mathbf{T}$ he incidence of ventricular tachycardia (VT) in childhood is low $(0.2-0.8$ per 10,000 children in school-based heart screening) compared with that in adults ${ }^{1-6}$ in whom most VT is associated with coronary artery disease. In contrast, most pediatric cases are idiopathic without underlying heart disease and are found incidentally during school-based heart screening, with the exception of those found as complications of congenital heart diseases. The aim of the present study was to review the clinical picture and long-term course of patients in whom VT was detected by school-based screening.

\section{Methods}

\section{Subjects}

The subjects were 48 children (23 males, 25 females) diagnosed with VT after routine school-based heart examinations conducted before they entered school or while they attended primary, junior or senior high school. All children undergo a 12-lead ECG, which pediatric cardiologists or

(Received October 6, 2004; revised manuscript received December 1, 2004; accepted December 16, 2004)

Cardiovascular Center, *Department of Paediatrics, Yokohama City University Medical Center, **Department of Paediatrics, Yokohama City University School of Medicine, Niimura Clinic and Thibata Clinic, Yokohama, Japan

Mailing address: Mari Iwamoto, MD, Cardiovascular Center, Yokohama City University Medical Center, 4-57 Urafune-cho, Minami-ku, Yokohama 232-0024, Japan. E-mail: maridelsol@ dream.com pediatricians then check for abnormalities (the rate of abnormal ECGs is approximately 2-3\% per annum). Children who have an abnormal ECG and have not been previously followed for heart disease, have a more detailed examination at a regional hospital. The present subjects were detected in this way and referred to the Department of Paediatrics, Yokohama City University Hospital where they were diagnosed as having VT. Of the 48 patients, underlying heart disease was detected in 2 cases (nonischemic left ventricular aneurysm, 1 ; arrhythmogenic right ventricular cardiomyopathy, 1), either at the time of the first medical examination after school-based screening or during follow-up. All of the 48 patients were followed for more than 2 years (2-30 years; average: 6 years) after the medical examinations. At the first medical examination, these subjects ranged in age from 5 to 15 years (average: 11 years).

\section{Cardiac Examinations}

All subjects underwent an examination consisting of a 12-lead ECG, treadmill exercise test, chest X-ray and echocardiography to determine the presence or absence of underlying heart disease. The site of origin and type of VT (monomorphic or polymorphic) were determined on the basis of the QRS morphology of the VT detected by 12lead ECG. Holter monitoring was used to record the (1) total number of premature ventricular contractions (PVC) in 1 day, (2) number of episodes of VT/day, the maximum number of salvos of VT and the heart rate during VT. Treadmill exercise stress testing and 24-h ECG monitoring 


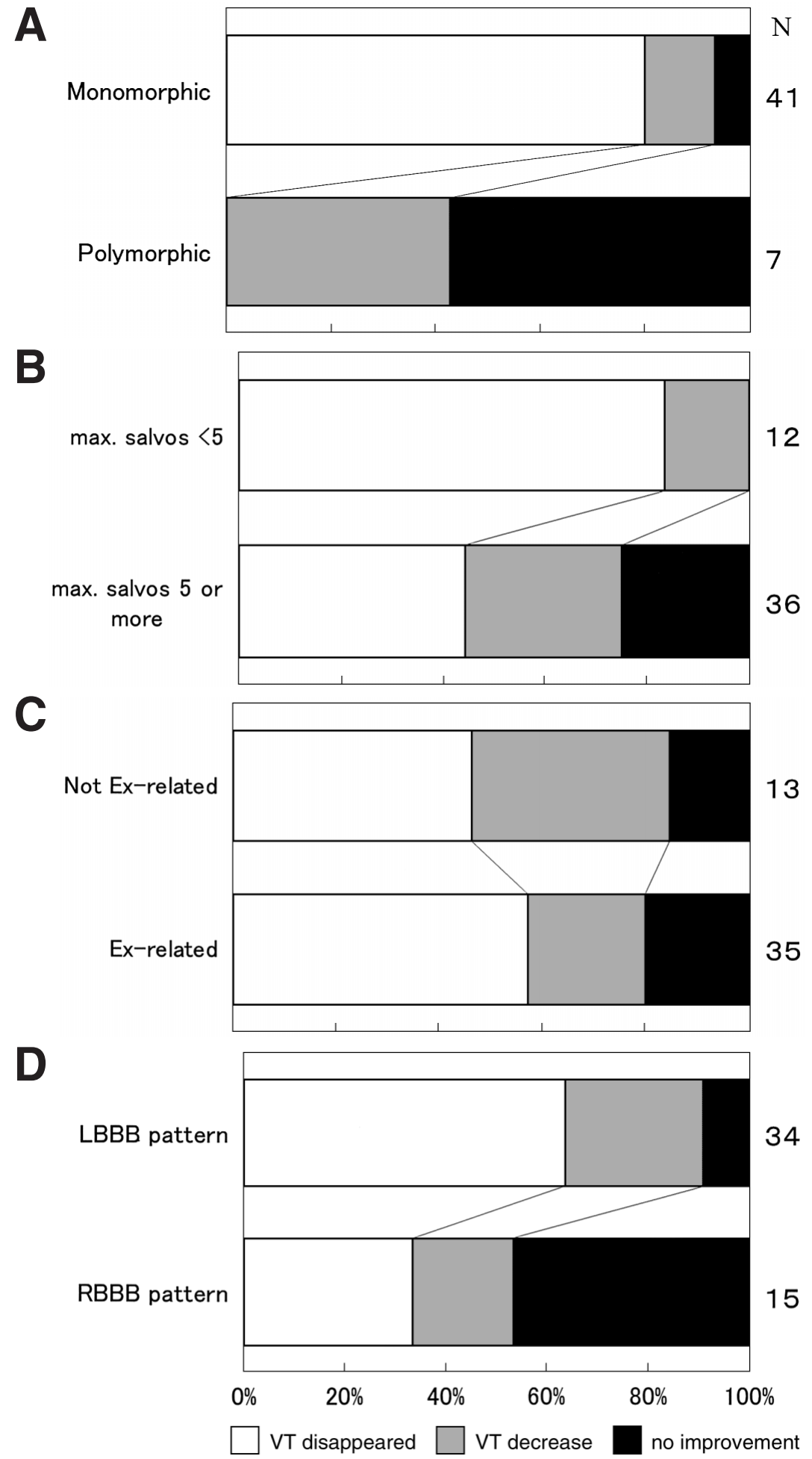

Fig 1. Long term course of ventricular tachycardia (VT). (A) Comparison of monomorphic and polymorphic VT, (B) comparison of VT with a maximum number (max.) of premature ventricular contraction salvos $<5$ or $>5$, (C) comparison of exercise (Ex)-related VT and nonexercise-related VT, (D) comparison of VT with a QRS morphology of LBBB or RBBB. were performed at 1-month intervals shortly after diagnosis, and every 6 or 12 months as follow-up. Antiarrhythmic agents were administered to 26 cases because the VT was fast or long or accompanied by symptoms and the medications were continued for at least 1 year. When VT did not recur during therapy, the drug doses were tapered off. We defined non-sustained VT as lasting from 3 beats to $30 \mathrm{~s}$ and terminating spontaneously, and sustained VT was defined as lasting more than $30 \mathrm{~s}$.

\section{Results}

Diagnosis

In 8 of the 48 cases (17\%), VT was detected by resting ECG at the time of the first examination at school and of the remaining 40 cases, $38(82 \%)$ were judged as having a single PVC, 1 as having first-degree atrioventricular block and 1 with ST depression. In these cases detection of VT required exercise stress ECG or Holter monitoring at the 
follow-up examination. The VT was induced by exercise stress in 33 cases, and was detected in 41 cases with 24-h ECG monitoring. By combining both methods, 47 cases were diagnosed as having VT at the first medical examination after the school-based screening. In the remaining case, VT was detected only after the second exercise stress test.

\section{Types of $V T$}

Of the 48 cases of VT, 3 were the sustained type and 45 the non-sustained type; 4 cases were symptomatic (syncope, 2; chest pain 2) and of these patients, 3 had polymorphic non-sustained VT, 2 of which had underlying heart disease, and 1 had sustained VT. The remaining cases were asymptomatic. The majority of patients (ie, 35) had exerciseinduced VT (non-sustained type, 33; sustained type, 2). As to the QRS morphology of the VT, 34 had a left bundlebranch block (LBBB: 28 had the inferior axis pattern) and 15 cases had a right bundle-branch block (RBBB). Monomorphic VT occurred in 41 cases and 7 cases were polymorphic VT, of which 5 had 2 or more RBBB patterns of the QRS morphologies of the VT, 1 had 2 patterns of LBBB, and 1 had both LBBB and RBBB patterns. Analysis of the Holter monitor recordings revealed a wide variation in the number of PVCs observed in 1 day, the values being $50-70,000 /$ day (average 24,800 $\pm 16,100$ ). However, in $80 \%$ of cases the number exceeded $10,000 /$ day. The number of episodes of VT ranged from 1-1,250/day (average: $268 \pm 311$ ) and in $50 \%$ of cases, there were more than 100 episodes/day.

\section{Treatment}

In this study, 26 patients received antiarrhythmic drugs: 12 cases had a heart rate greater than 180 beats/min at the time of VT, 10 cases had more than 10 salvos of VT, and 4 cases had symptoms such as attacks of syncope, palpitation, and shortness of breath. The therapeutic regimens used were $\beta$-blockers (13 cases), verapamil (4 cases), mexiletine ( 2 cases), $\beta$-blockers + verapamil ( 3 cases) and $\beta$-blockers + mexiletine ( 4 cases). The VT disappeared or decreased significantly (decrease in VT episodes of more than $90 \%$ on Holter monitoring) in 20 cases after drug administration, but in the remaining 6 cases, the VT was resistant to drug therapy.

\section{Long-Term Course}

The VT disappeared in 26 cases over a period of 1-7 years (average: $3.2 \pm 1.9$ years); in 9 cases, it was less than 2 years. The VT was monomorphic in all 26 cases and the QRS morphology most frequently seen was LBBB inferior axis pattern ( 19 of the 26 cases). In the other 22 cases the VT persisted, but the number of episodes per day showed a definite tendency to decrease by more than $90 \%$ during the course of follow-up in 8 of these cases. In contrast, no significant decrease was observed in any of the 4 symptomatic cases, in the patients with underlying disease (2 cases) or in any cases of polymorphic VT (7 cases).

Fig 1 shows the prognosis of VT in relation to the type of VT. Although monomorphic VT disappeared in $80 \%$ of cases over time, all cases of polymorphic VT were persistent.

VT disappeared in $83 \%$ of cases with a maximum number of 4 or less, where as if the maximal number of salvos was 5 or more, VT disappeared in $44 \%$ of cases, and in $25 \%$ of such cases there was no improvement, indicating a significant difference according to the number of salvos.

VT disappeared in approximately $60 \%$ of cases with a QRS morphology of LBBB, and in $38 \%$ of cases with RBBB, which suggests that the prognosis of VT differs according to its origin. There was no significant difference in the disappearance of VT between cases that were or were not exercise-related.

Underlying Heart Disease In one case suspected of having arrhythmogenic right ventricular cardiomyopathy, the VT was polymorphic and resistant to therapy. No abnormality was detected by echocardiography at the time of the first medical examination conducted when the patient was 15 years old, but enlargement of the right ventricular cavity became apparent over the course of 7 years. The other case had a non-ischemic left ventricular aneurysm, and the VT was thought to be of the exercise-induced nonsustained type with its origin near the aneurysm. The heart rate during VT and the number of salvos decreased after administration of $\beta$-blockers and the patient was advised to restrict exercise and continue with the medication and follow-up. Sudden death occurred 10 years later when the patient ceased taking medication.

\section{Discussion}

Most of the present cases were diagnosed as having PVC on resting ECG recorded during the first school-based heart screening and it was only with further medical examinations (exercise stress test or Holter monitor) that they were diagnosed as having VT. VT at a very young age is mostly exercise-related, $1,2,5,8-10$ as were $70 \%$ of the present cases, so an exercise stress ECG is indispensable for diagnosing the severe forms. An exercise stress ECG was performed in all cases of PVC, but Holter monitoring was sometimes omitted because it is time-consuming and costly even though it improves the rate of diagnosis of VT. The test is necessary to detect salvos, polymorphisms and frequent occurrence of PVC.

In the present study, VT was not detected in 14 of the 48 cases by further medical examination or exercise stress ECG. Of these, 12 cases were unrelated to exercise and had frequent occurrence of VT (total number: 10,000 $70,000 /$ day), and the remaining 2 cases were exerciserelated and detected by the second exercise stress test.

Almost all cases of VT detected by school-based screening in both the present patients and others are the idiopathic/non-symptomatic type without underlying heart disease, the prognosis of which is generally good1,3-17 After following 46 cases of idiopathic VT in childhood for an average period of 84 months, Tsuji et al reported that VT disappeared in $65 \%$ of cases; even the PVC disappeared and sinus rhythm was regained in $37 \%$ ? Pfammatter et al found that VT disappeared in $56 \%$ of 71 cases of idiopathic VT in children aged 1 year and older after an average period of observation of 46 months? In the present patients the VT resolved in $55 \%$ after an average period of 3.2 years, excluding the 2 cases with organic heart disease. In $27 \%$, the PVC disappeared and sinus rhythm was re-established. The mechanism of spontaneous disappearance is unclear. However, because VT reappeared in 2 patients after a lapse of 5-10 years after establishment of sinus rhythm, it is necessary to continue ambulatory observation at regular intervals for a long period of time? 


\section{Treatment Policy}

For asymptomatic cases of idiopathic monomorphic VT when the maximum number of salvos is small, observation of the course of disease is necessary, but treatment is not essential. ${ }^{15}$ We began treatment in 26 cases; for 16 cases of idiopathic VT with the origin in the outflow tract of the right ventricle, $\beta$-blockers were effective in 11 cases, and verapamil was effective in 5 cases.

Catheter ablation has been indicated for idiopathic VT in adults because of its good outcome ${ }^{17-19}$ and if the pediatric patient is of sufficient size, catheter ablation may be the choice of treatment for VT with its origin in the outflow tract of the right ventricle or VT with RBBB left axis deviation and its origin in the left ventricle. However, the longterm prognosis of catheter ablation is unknown with regard to ventricular muscle growth in patients who are still growing. If the patients are small $(<25 \mathrm{~kg})$, the need for general anesthesia, as well as the fact that more than 50\% of cases of idiopathic VT disappear spontaneously, it should be seriously considered whether catheter ablation is indicated.

In cases of polymorphic VT or VT with underlying heart disease, the VT persists for a long period. The tachycardia is mostly symptomatic and the risk of transition to ventricular fibrillation and sudden death is high?2,21 Furthermore, in not a few cases it is difficult to control the tachycardia with antiarrhythmic drugs and in such cases, administration of amiodarone or implantation of implantable cardioverter defibrillator should be considered.

\section{Conclusion}

The characteristic features of the long-term course of VT detected during school-based heart screening are as follows.

1. Most cases are idiopathic and non-sustained, and spontaneous disappearance occurs in more than $50 \%$ of cases over the course of time.

2. The prognosis is especially good with monomorphic VT when the maximum number of salvos is less than 5.

3. Many cases of polymorphic VT or cases with underlying organic heart disease are persistent, and some are resistant to treatment.

\section{References}

1. Rocchini AP, Chun PO. Ventricular tachycardia in children. Am J Cardiol 1981; 47: 1091-1097.
2. Bergdahl DM, Stevenson JG, Kawabori I, Guntheroth WG. Prognosis in primary ventricular tachycardia in the pediatric patient. Circulation 1980; 62: 897-901.

3. Tanabe T, Goto Y. Long-term prognostic assessment of ventricular tachycardia with respect to sudden death in patients with and without overt heart disease. Jpn Circ J 1989; 53: 1557-1563.

4. Fulton DR, Chung KJ, Tabakin BS, Keane JF. Ventricular tachycardia in children without heart disease. Am J Cariol 1985; 55: $1328-$ 1331.

5. Kawaide M, Aragaki T, Matsuda M, Oouchi H, Nakajima T, Kamiya T. Ventricular tachycardia in children. Iryou 1992; 46: 611-615 (in Japanese).

6. Tsuji A, Nagashima M. Long-term follow-up of idiopathic ventricular arrhythmias in otherwise normal children. Jpn Circ J 1995; 59: $654-662$.

7. Pfammatter JP, Thomas P. Idiopathic ventricular tachycardia in infancy and childhood. J Am Coll Cardiol 1999; 33: 2067-2072.

8. Niimura I, Shibata T, Minamisawa T. Exercise induced non-sustained ventricular tachycardia in young. Rinshyou Sports Igaku 1992; 9: 931-936 (in Japanese).

9. Lemery R, Burgada P. Nonischemic ventricular tachycardia: Clinical course and long-term follow-up in patients without clinically overt heart disease. Circulation 1989; 79: 990-999.

10. Deal B, Miller SM. Ventricular tachycardia in a young population without overt heart disease. Circulation 1986; 73: 1111-1118.

11. Kennedy HL, Whitlock JA. Long-term follow-up of asymptomatic healthy subjects with frequent and complex ventricular ectopy. $N$ Engl J Med 1985; 312: $193-197$.

12. Trappe HJ, Burgada P. Prognosis of patients with ventricular tachycardia and ventricular fibrillation: Role of the underlying etiology. J Am Coll Cardiol 1988; 12: 166-174.

13. Kinder C, Tamburro P, Koppo D. The clinical significance of nonsustained ventricular tachycardia: Current perspectives. Pacing Clin Electrophysiol 1994; 17: 637-670.

14. Busby MJ, Shefrin EA, FlegJL. Prevalence and long-term significance of exercise-induced frequent or repetitive ventricular ectopic beats in apparently healthy volunteers. J Am Coll Cardiol 1989; 14: $1659-1665$.

15. Ohnishi S, Kasanuki H. Long-term outcome of pharmacological and non-pharmacological treatment for ventricular arrhythmias. J Cardiol 2000; 35: 75-84.

16. Gaita F, Giustetto C, Di Donna P, Richiardi E, Libero L, Brusin MC, et al. Long-term follow-up of right ventricular monomorphic extra systoles. J Am Coll Cardiol 2001; 38: 364-370.

17. Klein LS, Miles WM. Ablative therapy for ventricular arrhythmias. Prog Cardiovasc Dis 1995; 37: 225-242.

18. Calkins H, Kalbfleisch SJ. Relation between efficacy of radiofrequency catheter ablation and site of origin of idiopathic ventricular tachycardia. Am J Cardiol 1993; 71: 827-833.

19. Tada Çg, Ito S, Naito S, Kuosaki K, Ueda M, Shinbo G, et al. Prevalence and electrocardiographic characteristics of idiopathic ventricular arrhythmia originating in the free wall of right ventricular outflow tract. Circ J 2004; 68: 909-914.

20. Viskin S, Belhassen B. Polymorphic ventricular tachyarrythmias in the absence of organic heart disease: Classification, differential diagnosis, and implications for therapy. Prog Cardiovasc Dis 1998; 41: 17-34.

21. Cho Y, Park T, Yang DH, Park HS, Chae J, Chae SC, et al. Arrhythmogenic right ventricular cardiomyopathy and sudden cardiac death in young Koreans. Circ J 2003; 67: 925-928. 